\title{
Distribuição espacial dos equipamentos públicos para comercialização de frutas, legumes e verduras em Curitiba, Paraná, Brasil
}

\author{
Spatial distribution of public outlets for fruit and vegetable sales \\ in Curitiba in the state of Paraná, Brazil
}

Aline Daniela da Cruz e Silva (https://orcid.org/0000-0003-1159-5196) ${ }^{1}$

Aichely Rodrigues da Silva (https://orcid.org/0000-0001-9447-2380) ${ }^{2}$

Doroteia Aparecida Hofelmann (https://orcid.org/0000-0003-1046-3319) ${ }^{1}$

${ }^{1}$ Programa de PósGraduação em Saúde Coletiva, Universidade Federal do Paraná. Rua Padre Camargo $2803^{\circ}$ Andar, Alto da Glória. 80060-240

Curitiba PR Brasil.

alinedaniela.w@gmail.com

${ }^{2}$ Secretaria Municipal de

Educação. Imperatriz MA

Brasil.
Abstract Greater availability of fruit and vegetables (FV) may enhance the consumption of these food products. This study sought to investigate demographic and household income inequalities in the spatial distribution of FV in the city of Curitiba, State of Paraná. A total of 106 FV outlets were listed in the 10 administrative regions on the City Hall Supply Department website. Data on population and household income of the administrative regions were obtained from the 2010 Demographic Census. The food sale outlets were identified, and subsequently analyzed for the correlation between the regions by the Moran index. A total of 407 food stands were evaluated in 103 public facilities that commercialized FV. A concentration of street food markets in the central areas of the city, with a global Moran index of 0.99 , was observed. In the regions, only one did not have an outlet (Tatuquara). There was a higher concentration of outlets in the higher-income regions (9.82/10,000 inhabitants) compared to those with the lower income regions $(2.60 / 10,000$ inhabitants) $(p<0.001)$. Inequities were observed in the supply and in the quality of $F V$ available in public facilities in lower-income regions, which should be considered by administrators when planning distribution in these locations.

Key words Healthy eating, Spatial distribution, Socioeconomic factors, Public policies
Resumo A maior disponibilidade de frutas, verduras e legumes (FLV) pode auxiliar no consumo destes alimentos. Objetivou-se investigar desigualdades demográficas e de renda domiciliar na distribuição espacial da comercialização de FLV em Curitiba, Paraná. Foram identificados 106 equipamentos de venda de alimentos listados no site da Secretaria Municipal de Abastecimento, nas 10 regionais administrativas do município. Os dados populacionais e de Renda Domiciliar das regionais administrativas foram obtidos do Censo Demográfico de 2010. Os pontos de comercialização de alimentos foram identificados e, em seguida, analisados quanto a correlação entre as regionais pelo indice de Moran. Foram avaliadas 407 barracas, em 103 equipamentos públicos que comercializavam FLV. Observou-se concentração das feiras na área central do município, com indice de Moran global de 0,99. Entre as regionais apenas uma não apresentou nenhum equipamento (Tatuquara). Observou-se concentração dos equipamentos nas regionais com maior renda $(9,82 / 10.000 \mathrm{hab}) \mathrm{em}$ relação àquelas com menor renda $(2,60 / 10.000$ hab) $(p<0,001)$. Existem iniquidades na oferta e na qualidade dos FLV em equipamentos públicos em regionais de menor renda, o que deve ser considerado pelos gestores ao programarem a distribuição dos locais.

Palavras-chave Alimentação saudável, Distribuição espacial, Fatores socioeconômicos, Políticas públicas 


\section{Introdução}

O aumento do consumo de alimentos ultraprocessados é uma das principais causas da pandemia de obesidade e Doenças Crônicas Não Transmissíveis (DCNT) ao redor do mundo ${ }^{1}$. A substituição de alimentos in natura por produtos prontos para consumo aconteceu nos países desenvolvidos durante o século $\mathrm{XX}$, e estendeuse aos países emergentes, com o crescimento da economia e das indústrias transnacionais. No Brasil, a presença de produtos ultraprocessados cresce exponencialmente, considerada uma das explicações para a tendência crescente de excesso de peso na população brasileira² ${ }^{2}$.

Por outro lado, o consumo de frutas e hortaliças é considerado um importante marcador para uma alimentação saudável, determinante para a saúde, e pode ser responsável pela diminuição no risco de $\mathrm{DCNT}^{3}$. A maioria da população mundial não atinge as quantidades recomendadas destes alimentos, que associados a outros fatores contribuem para a prevenção de doenças. Em 2018, no conjunto das 27 capitais brasileiras, apenas $33,9 \%$ da população adulta apresentou consumo de FLV cinco ou mais dias da semana. Essa ingestão foi menor em homens $(27,7 \%)$ do que em mulheres $(39,2 \%)^{4}$. As capitais que apresentaram maiores frequências de consumo regular foram Florianópolis (44,7\%), Belo Horizonte $(44,1 \%)$ e Curitiba $(42,2 \%)$. As piores frequências de consumo foram verificadas em Belém (23,0\%) e em Macapá $(25,0 \%)^{4,5}$. As capitais que apresentaram maiores frequências de consumo de FLV foram aquelas com alto Índice de Desenvolvimento Humano Municipal (IDHM), sendo 0,847, 0,810 e 0,823 , respectivamente. Em contrapartida, os municípios de Belém e Macapá tendo IDHM de 0,746 e 0,733 , respectivamente, sendo considerados valores de IDHM médio ${ }^{6}$. Estes achados reforçam as desigualdades no consumo de FLV de acordo com a renda, o que pode estar relacionado com piores condições de acesso e menor poder de compra da população, levando a uma diminuição na qualidade da dieta e aumentando as iniquidades socioeconômicas na saúde entre diferentes locais do país ${ }^{7-9}$.

O Guia Alimentar para a População Brasileira publicado em 2014 aponta a influência de diversos fatores nas escolhas alimentares, tais como: políticos, sociais, econômicos e ambientais, ressaltando que o ambiente é determinante nas escolhas alimentares ${ }^{3}$. O ambiente alimentar pode ser definido como o local onde se tem acesso aos alimentos, interferindo na qualidade da alimentação. Este pode facilitar ou dificultar as escolhas alimentares, dependendo das condições ofertadas $^{10,11}$. Locais com baixo acesso a uma alimentação adequada geralmente são áreas com maiores desigualdades sociais, distanciando essas populações do Direito Humano à Alimentação Adequada (DHAA) $)^{3,12}$.

A influência do ambiente sobre a alimentação mostra a necessidade de entender a dinâmica do uso do solo, da produção, distribuição, comercialização e abastecimento de alimentos saudáveis, e o desenvolvimento de políticas públicas intersetoriais, a fim de garantir a Segurança Alimentar e Nutricional (SAN) e o DHAA para todas as populações $^{8,13}$. Desde 2003, a fim de melhorar as condições de SAN em populações de menor renda, foi criada pela Secretaria Nacional de Segurança Alimentar e Nutricional e a Secretaria $\mathrm{Na}-$ cional de Renda e Cidadania, uma estratégia de criação e manutenção de Equipamentos Públicos de Alimentação e Nutrição, como Restaurantes Populares, Bancos de Alimentos e Programa de Aquisição de Alimentos e Feiras e Agricultura Urbana. A implantação destes equipamentos associada à melhoria nas condições de compra, pode favorecer a SAN na população ${ }^{14}$.

A investigação do ambiente enquanto influência no consumo alimentar é fundamental na atualidade, pois as relações entre alimentação e saúde são complexas. As informações referentes ao território podem possibilitar a criação de ambientes saudáveis, por meio de políticas públicas e estratégias em saúde ${ }^{15}$. No Brasil, estudos vêm sendo realizados objetivando avaliar essas relações. Na cidade de São Paulo (SP), por exemplo, verificou-se que áreas com maior concentração de feiras livres e sacolões apresentam maiores taxas de consumo de FLV entre adultos. Também foram encontradas discrepâncias na distribuição do comércio de alimentos: áreas com melhores condições socioeconômicas apresentaram maiores densidades de comércio de alimentos ${ }^{16}$.

Em Belo Horizonte (MG) observou-se um aumento no consumo de FLV à medida que a quantidade de equipamentos de comercialização destes alimentos era maior, assim como em áreas com maior concentração de renda. Os menores escores de consumo de FLV foram observados nas áreas com maior densidade de alimentos não saudáveis, demonstrando que a presença deste tipo de comércio pode contribuir para o ambiente obesogênico ${ }^{17}$. As feiras livres podem auxiliar na melhoria do ambiente, assegurando a oferta de alimentos com características locais, mais saudáveis e promover a agricultura familiar, geran- 
do ocupações de trabalho e renda, além de estar mais próximo da população ${ }^{18,19}$. A criação destes equipamentos públicos de comércio de alimentos, como as feiras, concebidos como instrumentos de regularização do varejo, pode reduzir a intermediação mercantil e tornar os preços mais acessíveis para a comunidade,sendo outra iniciativa capaz de melhorar o ambiente alimentar, principalmente em locais menos favorecidos economicamente ${ }^{20}$.

Nas últimas décadas observou-se um aumento significativo de estudos sobre as desigualdades sociais e o acesso a uma alimentação variada ${ }^{8,9,21,22}$. No Brasil, de acordo com uma metanálise sobre estudos relacionados à SAN, as populações com maior grau de pobreza apresentaram maiores chances de vulnerabilidades quanto à disponibilidade de alimentos ${ }^{23}$. Dessa forma, conhecer o ambiente alimentar em diferentes municípios brasileiros possibilitará auxiliar na formulação de políticas públicas que assegurem a SAN da população. O presente artigo objetivou verificar a existência de desigualdades demográficas e de renda domiciliar na distribuição espacial dos equipamentos públicos de venda de frutas, legumes e verduras do município de Curitiba, Paraná.

\section{Métodos e delineamento do estudo}

Trata-se de um estudo transversal, realizado no município de Curitiba, capital do Paraná, sendo este constituído por 75\% de área urbana. O município possuía população estimada de 1.917.185 pessoas em 2018, segundo o Instituto Brasileiro de Geografia e Estatística (IBGE). O município divide-se em 10 regionais administrativas que são: Bairro Novo, Boa Vista, Boqueirão, Cajuru, CIC, Matriz, Pinheirinho, Portão, Santa Felicidade e Tatuquara, com 75 bairros ao todo. Possui um Índice de Desenvolvimento Humano Municipal (IDHM) de 0,823, considerado muito alto (de 0,8 a 1), e uma Renda Média Domiciliar (RMD) de R\$3.774,19 em 2010 - valor acima da média nacional. Contudo, são observadas importantes desigualdades intramunicipais na distribuição da renda, por exemplo, o IDHM na regional Matriz era de 0,928, enquanto nas regionais Pinheirinho e CIC era de 0,753 e 0,755, respectivamente ${ }^{24}$.

\section{Coleta de dados}

A descrição do ambiente alimentar foi realizada por meio da quantificação dos pontos de venda de FLV registrados na Secretaria Munici- pal de Abastecimento (SMAB). Esses pontos de venda, referidos como Equipamentos Públicos, foram listados de acordo com o site da SMAB (http://www.curitiba.pr.gov.br/secretarias/equipamentos/abastecimento/9) e utilizados como base na criação de um banco de dados; utilizando nome, endereço, dia e horário de funcionamento. Durante toda a coleta, houve acompanhamento periódico do site, visando adicionar ou excluir equipamentos em caso de alterações no funcionamento.

Os critérios para inclusão dos equipamentos públicos na pesquisa foram: serem administrados pela SMAB, seguindo o Plano Municipal de Segurança Alimentar e Nutricional (PLAMSAN), e serem de livre acesso à população. Desta forma, equipamentos como os Armazéns Populares não foram inseridos no estudo. Os Equipamentos existentes são categorizados pela $\mathrm{SMAB}$ em oito tipos: Feira Livre, Feira Livre Noturna, Feira Gastronômica, Feira de Orgânicos, Mercados Públicos, Direto da Roça, Sacolão da Família e Programa Nossa Feira.O Sacolão da Família e o Programa Nossa Feira tem como objetivo ofertar alimentos subsidiados a um preço único, custando R\$2,29 por quilo, durante os anos de 2018 e 2019, obtido por meio da compra direta de agricultores da região para redução de custos e priorizando os produtos da safra ${ }^{25}$.

Os pontos dos equipamentos foram delimitados, por meio dos endereços disponibilizados pelo site da SMAB, em um aplicativo de mapas (Google Maps). Iniciou-se a coleta realizando a verificação dos endereços in loco. A coleta de dados em todos os equipamentos de venda descritos anteriormente deu-se de março a dezembro de 2018. Evitou-se realizar coleta em feriados e dias de chuva, para minimizar perdas por ausência dos comerciantes. Todas as pesquisadoras passaram por capacitação prévia para entendimento do instrumento e treinamento dos procedimentos da pesquisa.

Os procedimentos de coleta consistiam em: listagem de todas as barracas existentes em cada equipamento, registro do número de barracas e do tipo de produto ofertado em cada uma; explicação da pesquisa ao responsável pela barraca e apresentação do Termo de Consentimento Livre e Esclarecido e aplicação do instrumento. Os equipamentos do tipo "preço único" foram contabilizados como uma barraca cada, pois o ponto de venda é único.

Para avaliação do acesso aos equipamentos de venda de alimentos utilizou-se o instrumento de avaliação do acesso a alimentos para consumo 
no domicílio (HFSI), proposto por Duran. O instrumento consiste em um questionário, do tipo check-list, que varia de 0 a 15 pontos e mede a disponibilidade, variedade e propaganda de FLV, presença de vendas ao "preço fixo" e disponibilidade de alimentos ultraprocessados. A presença de itens relativos à uma alimentação saudável e a presença de alimentos orgânicos pontuam positivamente; $\mathrm{e}$ a presença ou publicidade de alimentos ultraprocessados, negativamente. Desta forma, as maiores pontuações indicam melhor qualidade de acesso aos equipamentos. Essa pontuação permite uma comparação entre as feiras, demonstrando a qualidade da oferta dos alimentos ${ }^{26}$.

\section{Tratamento e análise dos dados}

A delimitação das regionais seguiu os bancos de dados do Instituto de Pesquisa e Planejamento Urbano de Curitiba. A partir dos endereços dos equipamentos públicos, foi realizado o georreferenciamento e construção de mapas temáticos, com auxílio do software QGIS 2.18 Las Palmas.

Para observação dos indicadores socioeconômicos e demográficos foram utilizadas as informações referentes à área, população, densidade demográfica e RMD das regionais de Curitiba, obtidas a partir de dados secundários, do Instituto de Pesquisa e Planejamento Urbano de Curitiba (http://www.ippuc.org.br/\#), a partir do Censo Demográfico de 2010.

A densidade dos pontos de venda de FLV nos equipamentos públicos foi calculada dividindose o número de feiras para cada 10.000 habitantes das regionais. As diferenças na densidade de pontos de venda em relação aos tercis da RMD foram investigadas por meio do teste de tendência linear.

Esses dados foram representados com a utilização do estimador de densidade Kernel (mapa de calor). A estimação da densidade Kernel é uma técnica de interpolação apropriada para posições de dados individuais ${ }^{27}$. Essa análise foi realizada com o software QGIS. Além disso, para demostrar a área de abrangência dos equipamentos públicos foram criados raios (buffers) com a área de influência de 1,6 quilômetros, com auxílio do complemento MMGIS (http://michaelminn. com/linux/mmqgis/) no software QGIS. O buffer de $1,6 \mathrm{~km}$ foi escolhido baseado na literatura, sendo considerada uma distância possível de ser percorrida pelos consumidores para compra dos alimentos $^{26,28-30}$.

Por fim, para constatar a autocorrelação espacial entre as regionais, aplicou-se o índice de
Moran global I e Moran Local (LISA). A autocorrelação espacial considerou as regionais com valores de índice semelhantes, o Moran I o conjunto dos dados por regional, enquanto o LISA forneceu um valor para cada regional, bem como os agrupamentos para os indicadores selecionados. O índice de Moran indica que - 1 o agrupamento de valores dissimilares; 0 não é autocorrelação; +1 indica agrupamento de valores semelhantes. Para o Moran (LISA) fui utilizada a função K de Ripley do vizinho mais próximo na análise.

As correlações consideraram o nível de significância $\mathrm{p} \leq 0,05$, foram classificadas como "alto -alto" mostram as regionais com altas proporções do indicador e "baixo-baixo" para as regionais que não possui correlação com as demais regionais quanto a comercialização de frutas, verduras e legumes. Para essa análise foram utilizados os software Geoda e QGIS.

\section{Aspectos éticos}

A avaliação dos equipamentos públicos foi submetida como adendo ao projeto intitulado "Excesso de peso e características do ambiente escolar em estudantes de Curitiba, Paraná, e aprovada pelo Comitê de Ética em Pesquisa em Seres Humanos do Setor de Ciências da Saúde, da Universidade Federal do Paraná.

\section{Resultados}

No momento do levantamento de dados da pesquisa, em março de 2018, existiam 106 equipamentos públicos de comercialização de alimentos listados no site da SMAB. Porém, durante a coleta, dois destes equipamentos não foram encontrados, sendo ambos localizados na regional Tatuquara; e um dos equipamentos não ofertava FLV, apenas pescado, e estava localizado na regional Matriz. Dentre todos os 103 equipamentos, foram contabilizadas 1.072 barracas, sendo 407 $(38,0 \%)$ de FLV. Houve 13 recusas de participação, sendo duas em barracas de feiras livres e 11 em mercados. Sendo assim, 394 barracas (36,8\% do total) foram incluídas no estudo.

Os equipamentos do tipo Feira Livre Volante estavam em maior quantidade $(\mathrm{n}=38 ; 37 \%)$, seguidos pelas Feiras de Orgânicos $(n=15 ; 15 \%)$ e pelas Feiras Livres Noturnas e os Sacolões da Família ( $\mathrm{n}=14 ; 14 \%$, cada). O Programa Nossa Feira apresentou 10 pontos de venda (10\%), os equipamentos Direto da Roça e Mar estavam em 7 pontos $(7 \%)$, porém um destes se resumia 
a uma barraca de pescados. Por fim, em menor quantidade, estavam as Feiras Gastronômicas (n $=3 ; 3 \%)$ e os Mercados $(n=2 ; 2 \%)$.

A distribuição das características das regionais é apresentada na Tabela 1. Tatuquara (R\$1.674) e Bairro Novo ( $\$ 2.013)$ apresentaram as RMD mais baixas, enquanto Matriz (R\$6.439) e Portão ( $\mathrm{R}$ \$5.297) os maiores valores da RMD.As regionais foram então distribuídas em tercis de RMD: $1^{\circ}$ tercil de $\mathrm{R} \$ 1.674$ a $3.261,2^{\circ}$ tercil de $\mathrm{R} \$ 3.262$ a 4.850 , e $3^{\circ}$ tercil de $\mathrm{R} \$ 4.851$ a R\$6.439. As regionais Boa Vista, CIC e Santa Felicidade foram aquelas com maior extensão territorial, enquanto Portão, Cajuru, Pinheirinho e Matriz foram aquelas com maior densidade demográfica. O número de barracas de FLV nas regionais variou de 229 na regional Matriz a 0 na regional Tatuquara. $\mathrm{O}$ índice de acesso variou de 0 na regional Taquara a 12 na Matriz (Tabela 1 ).

A Figura 1 mostra a densidade de pontos de venda de FLV. A densidade total de barracas no município foi de 0,24 barracas $/ \mathrm{km}^{2}$. Quanto maior a renda da regional, maior a densidade de barracas. A oferta de FLV convencionais foi 4,2 vezes maior nas regionais localizadas no tercil de maior renda $\left(2,96 / 10.000\right.$ hab. $3^{\circ}$ tercil $)$ comparada àquele de menor renda $(0,70 / 10.000$ hab. $1^{\circ}$ tercil). A oferta de FLV orgânicos foi quatro vezes menor que a de convencionais, no tercil de maior renda. Nos outros tercis de RMD, a oferta de orgânicos foi quase nula. Em relação à oferta de alimentos dos equipamentos tipo "preço único", o primeiro e o segundo tercil tiveram maior densidade que o terceiro tercil $\left(0,15-1^{\circ}\right.$ tercil; $0,24-2^{\circ}$ tercil; e $0,06-3^{\circ}$ tercil) (Figura 1 ).
A área quente identificada pela análise do estimador de Kernel, representando a maior densidade de pontos de venda de FLV, localizou-se na região central do município e consequentemente nas regionais de maior RMD (regionais Matriz e Portão). À medida que ocorre um distanciamento da região central da cidade (Matriz), observase uma redução da concentração dos equipamentos que comercializavam FLV. As regionais com menor RMD apresentaram menores concentrações de pontos de venda, destacando-se uma regional (Tatuquara) com nenhum equipamento público de venda de alimentos (Figura 2).

A concentração dos equipamentos na região central do município foi confirmada pelo teste de Moran global, com valor de 0,99 . Isto sugere que bairros vizinhos dentro das regionais apresentam maior semelhança quanto ao padrão de distribuição dos equipamentos. Adicionalmente, o Moran local indicou existência de correlação espacial do tipo alto (FLV) -alto (RMD) na regional Matriz (índice de Moran local 1,31, $\mathrm{p}=0,001)$. Por outro lado, as regionais de pior RMD (Tatuquara e Bairro Novo) apresentaram correlação do tipo baixo-baixo, ou seja, baixa oferta de FLV e baixa RMD (1-3; índice de Moran local0,30 e 0,39) p = $0,004 \mathrm{e} 0,001$, respectivamente), enquanto na regional Boa Vista, observou-se correlação do tipo baixa disponibilidade de FLV e alta renda (índice de Moran local -0,14, p=0,039) (Figura 3).

Em relação ao índice de qualidade das feiras, o Moran global teve valor de 0,31 , indicando a presença de correlação espacial positiva, sugerindo que existe um padrão espacial na qualidade dos equipamentos entre as regionais. O Moran

Tabela 1. Distribuição das características socioeconômicas das regionais do município de Curitiba, Paraná, 2018.

\begin{tabular}{lccccccc}
\hline \multicolumn{1}{c}{ Regional } & Área $\left(\mathbf{K m}^{2}\right)$ & $\begin{array}{c}\text { População } \\
(\mathbf{h a b})\end{array}$ & $\begin{array}{c}\text { Densidade } \\
\left(\mathbf{h a b} / \mathbf{K m}^{2}\right)\end{array}$ & Renda $(\mathbf{R} \mathbf{})$ & FLV & $\begin{array}{c}\text { Densidade } \\
\text { FLV } \\
\left(\mathbf{b a r r a c a s K m}^{2}\right)\end{array}$ & $\begin{array}{c}\text { Índice } \\
\text { Acesso }\end{array}$ \\
\hline Bairro Novo & 45,01 & 145.433 & $3.231,25$ & $2.012,76$ & 1 & 0,1 & 10 \\
Boa Vista & 62,51 & 248.698 & $3.978,47$ & $3.727,49$ & 27 & 1,1 & 12 \\
Boqueirão & 39,81 & 197.346 & $4.957,62$ & $2.837,22$ & 15 & 0,8 & 11 \\
Cajuru & 35,76 & 215.503 & $6.025,55$ & $3.133,99$ & 17 & 0,8 & 11 \\
CIC & 63,46 & 184.482 & $2.907,09$ & $2.148,14$ & 1 & 0,1 & 8 \\
Matriz & 35,93 & 205.722 & $5.725,33$ & $6.438,71$ & 229 & 11,1 & 12 \\
Pinheirinho & 24,71 & 147.528 & $5.969,75$ & $2.882,5$ & 4 & 0,3 & 11 \\
Portão & 24,37 & 179.155 & $7.351,61$ & $5.297,41$ & 68 & 3,8 & 12 \\
Santa Felicidade & 62,22 & 146.081 & $2.347,88$ & $4.823,93$ & 26 & 1,8 & 13 \\
Tatuquara & 41,03 & 81.959 & $1.997,56$ & $1.674,84$ & 0 & 0,0 & 0 \\
\hline
\end{tabular}

Legenda: Dados de Área, População, Densidade e Renda - Censo Demográfico, 2010; FLV - Frutas, Legumes e Verduras. 


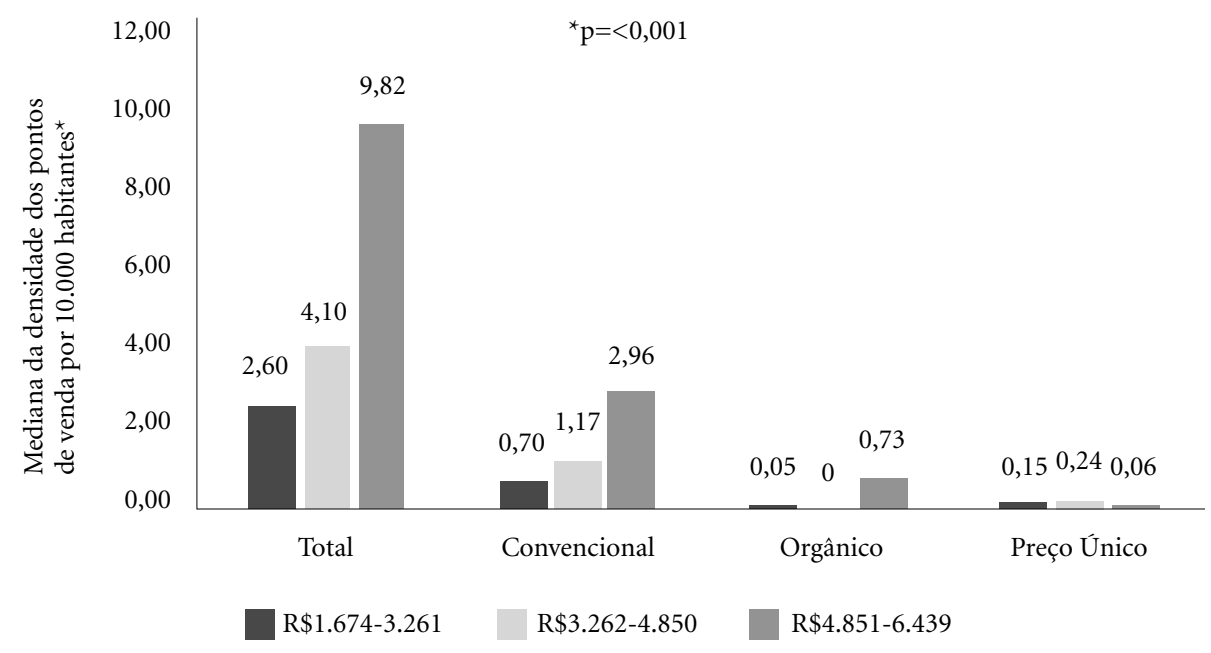

Figura 1. Densidade de pontos de venda de frutas, legumes e verduras nos equipamentos públicos de venda de alimentos de acordo com os tercis da renda das regionais administrativas de Curitiba, Paraná, 2018.

*Tercis de renda e densidade dos pontos de venda nas regionais, Teste de Tendência Linear

Fonte: Elaborado pelos autores.

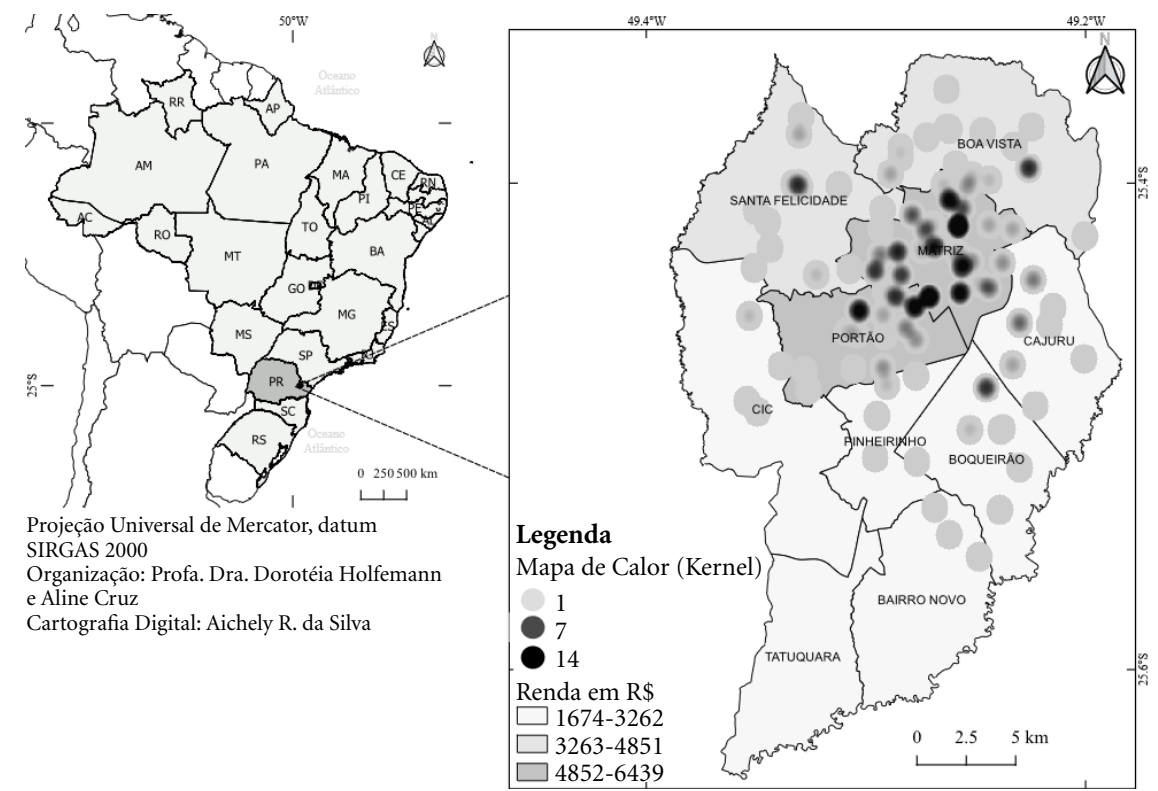

Figura 2. Mapa de Kernel da concentração dos pontos de venda de frutas, legumes e verduras de acordo com a Renda Média Domiciliar das regionais do município de Curitiba, Paraná, 2018.

Fonte: Elaborado pelos autores. 


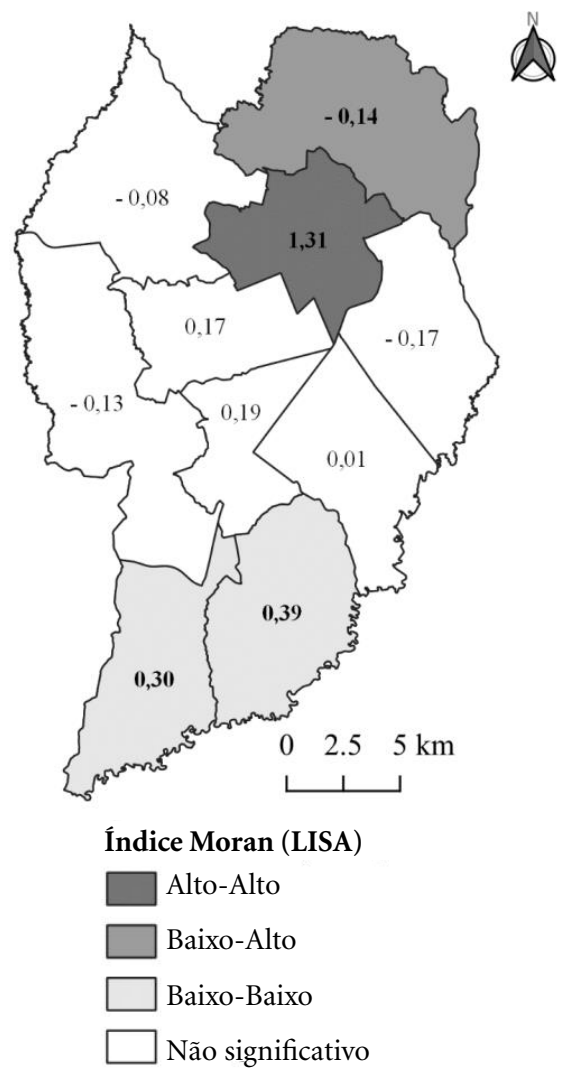

Figura 3. Índice de Moran local (LISA) de pontos de venda de frutas, legumes e verduras e Renda Média Domiciliar de Curitiba, Paraná, 2018.

Legenda: Valores significativos $\mathrm{p} \leq 0,05$ destacados em negrito, dados pelo índice de Moran local (LISA).

Fonte: Elaborado pelos autores.

local indicou correlação espacial do tipo alto -alto, ou seja, alto índice de acesso e alta RMD nas regionais Matriz (índice de Moran local0,19, $\mathrm{p}=0,001$ ) e Boa Vista (índice de Moran local 0,32, $\mathrm{p}=0,034)$; a regional Bairro Novo apresentou alto índice de acesso e baixa renda (índice de Moran local $-0,28, p=0,001$ ), enquanto a regional Tatuquara apresentou correlação do tipo baixo-baixo(índice de Moran 1,63, p=0,008). (Figura 4).

\section{Discussão}

O presente estudo revelou que os equipamentos públicos de venda de FLV estão concentrados na região central do município, sendo estas as áreas com maiores RMD. Nestas áreas, a oferta destes alimentos é maior que em áreas com menor RMD. Os equipamentos do tipo "preço único" apresentaram maior concentração nas áreas com menor RMD. A magnitude das diferenças é maior para feiras orgânicas. As piores condições de acesso foram encontradas nas regionais localizadas nas regiões periféricas, com menor renda. A utilização da geoestatística e do uso de ferramentas de geoprocessamento contribuíram para a compreensão da temática analisada e sua distribuição espacial nas regionais ${ }^{31,32}$.

Outros municípios demonstraram o mesmo perfil de distribuição desses pontos de venda de FLV. Em Jundiaí (SP), a maior densidade de equipamentos de varejo de FLV estava em áreas com maior renda(10). Em Florianópolis (SC), áreas com menor renda apresentaram menor densidade de comerciantes de alimentos, do que em áreas com rendimentos médios e elevados. Neste estudo, ao categorizarem os alimentos em: saudáveis, não saudáveis e mistos, a oferta de alimentos saudáveis era três vezes maior em áreas de maior renda ${ }^{15}$.

Acessibilidade geográfica, que se traduz em proximidade da residência ou do trabalho, são fatores que determinam o acesso ao alimento ${ }^{33}$. Em Belo Horizonte (MG) verificou-se que as pessoas que moravam em bairros com maior densidade de estabelecimentos apresentaram maior consumo de $\mathrm{FLV}^{17}$. O presente estudo verificou uma concentração de equipamentos de venda destes alimentos no centro do município, indicando que uma parcela da população pode encontrar dificuldade no acesso e assim ter uma diminuição no consumo. Por outro lado, a disponibilidade destes equipamentos em áreas centrais, pode torná-los disponíveis a partir dos deslocamentos no trajeto casa/trabalho.

A facilitação no acesso aos vegetais está associada à melhoria no consumo e, consequentemente, à melhoria nas condições de saúde da população. Em um estudo observacional, verificou-se que a disponibilidade de FLV esteve associada com a presença destes alimentos nas refeições familiares ${ }^{33}$. Outro estudo, que implementou mercados em áreas desabastecidas de comércio de FLV, verificou que, após a intervenção, os moradores destas localidades demonstraram aumento significativo no consumo destes alimentos $^{34}$.

Além da facilitação do acesso, em diferentes regiões do Brasil estudos demonstram que a renda familiar é a variável que se apresenta, na maioria das vezes associada significativamente à insegurança alimentar ${ }^{23}$. As pessoas com menor 

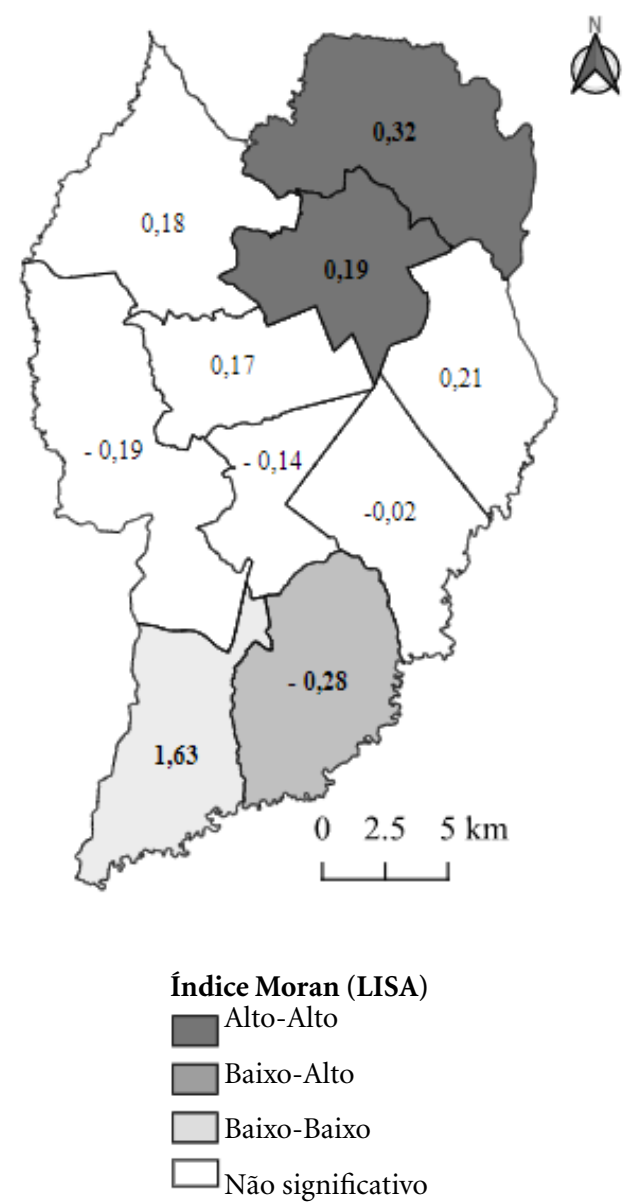

Figura 4. Índice de Moran local (LISA) da relação entre índice de qualidade dos equipamentos e Renda Média Domiciliar de Curitiba, Paraná, 2018.

Legenda: Valores significativos $\mathrm{p} \leq 0,05$ destacados em negrito, dados pelo índice de Moran local (LISA).

Fonte: Elaborado pelos autores.

renda têm o consumo alimentar caracterizado por alimentos de alta densidade energética, ricos em açúcares e gorduras, e não conseguem comprar em quantidade suficiente alimentos considerados saudáveis, como FLV, em comparação as pessoas com melhor renda. Isso irá refletir nas condições de saúde destes indivíduos, levando-os a diversos problemas relacionados à baixa qualidade da dieta ${ }^{15,33,35}$.

Da mesma forma que as áreas mais centrais apresentaram maior oferta de FLV, é provável que, pela maior quantidade de comércio, exista uma grande oferta de alimentos ultraprocessados também nesta área, porém o presente estudo focou na oferta de FLV nos equipamentos públicos. Em áreas de menor renda, é maior a presença de pequenos mercados e padarias, que ofertam em sua maioria, alimento processados e ultraprocessados, favorecendo o consumo destes alimentos e contribuindo para uma dieta obesogênica ${ }^{10,36}$.

Em Curitiba, um estudo que avaliou a distribuição do ambiente para atividade física nas escolas encontrou piores condições do ambiente em regiões de menor renda e mais distantes do centro, reforçando a ideia de que as periferias das cidades apresentam maiores iniquidades, e por consequência, reforçam um ambiente obesogênico ${ }^{37}$. Entender as características do município torna-se então indispensável para um planejamento de políticas públicas que auxiliem essa população a melhorar suas condições de saúde.

Dentre as regionais de Curitiba, a Matriz foi aquela que apresentou a maior concentração de equipamentos. Esta regional, tem os bairros mais antigos da cidade, incluindo o Centro, e apresenta características singulares quanto à sua distribuição demográfica, com uma maior proporção de adultos e idosos. Com maior oferta de empregos e estabelecimentos comerciais, é a regional mais desenvolvida, justificando a maior renda do município. Por outro lado, a regional Tatuquara tem grande concentração de indústrias, justificando a menor densidade populacional do município. Porém foi a regional com maior variação populacional entre 2010 e 2016, e demonstra grande dependência socioeconômica e dificuldades no acesso a serviços e equipamentos públicos ${ }^{37}$.

As evidências de que a proximidade com a oferta de alimentos saudáveis auxilia o seu consumo, assim como a proximidade de alimentos de alta densidade energética e baixo valor nutricional se associam a uma dieta de baixa qualidade estão crescendo rapidamente. Além disso, estudos demonstram que comunidades de baixa renda tem menor acesso à pontos de venda de alimentos saudáveis, e a qualidade dos alimentos ofertados tendem a ser piores nessas localidades $^{33-35}$. Nos Estados Unidos, um estudo com beneficiários de assistência governamental relataram que as maiores barreiras para o consumo de FLV foram o custo e a falta de tempo para preparo dos alimentos. Por outro lado, o acesso facilitado à FLV cultivadas localmente foi um fator facilitador para o consumo ${ }^{38}$. Outro estudo, também nos Estados Unidos, verificou que o acesso de FLV por famílias com crianças em SAN muito baixa era menor quando comparadas às famílias em SAN ${ }^{39}$. 
Para melhorar este quadro, em regiões de menor renda faz-se importante a implantação de políticas de SAN. Em um estudo realizado por Claro e Monteiro verificou-se que o aumento na renda das famílias mais pobres e a redução dos preços de FLV seriam importantes no aumento do consumo destes alimentos pelas famílias brasileiras $^{40}$. A utilização dos pontos de venda do tipo "preço único", com subsídio governamental, pode ser um incentivo para compra destes alimentos. Em Curitiba, os Sacolões da Família e o Programa Nossa Feira, seriam uma forma de redução de preço destes alimentos - mais baixos que os preços dos mercados tradicionais e provenientes de agricultores da região, portanto, mais acessíveis à população com menor renda ${ }^{25}$. Segundo a Secretaria de Abastecimento de Curitiba, busca-se ampliar o acesso e o consumo de FLV em todas as regiões do município, prevendo no Plano Municipal de Segurança Alimentar e Nutricional (PLAMSAN) ações como a implantação de pontos de venda ou subsídio a comerciantes de FLV, para maior alcance deste comércio ${ }^{41}$.

De acordo com o PLAMSAN, os programas de comercialização de alimentos têm por objetivo ampliar o acesso à FLV, sendo implantados em locais de fácil acesso à população e especialmente onde há maiores índices de pobreza e vulnerabilidade social ${ }^{41}$. Contudo, o estudo verificou que existe um desabastecimento das regionais de menor RMD, apresentando menor oferta de equipamentos públicos de venda de alimentos e, especificamente destes tipos de equipamentos públicos de preço único, essas áreas não são contempladas. Em Belo Horizonte (MG), os sacolões municipais também não estavam distribuídos aleatoria- mente, concentrando-se nas regiões com maiores IDHM do município, e a regional com baixo IDHM não contava com nenhum equipamento deste tipo ${ }^{8}$.

Este estudo teve como ponto forte o mapeamento da venda de FLV não apenas pela quantidade de equipamentos no município, mas quantificando a oferta por número de barracas de cada equipamento, o que fornece dados mais precisos sobre o acesso destes alimentos, bem como avaliação do índice de acesso, o qual permite inferir sobre a qualidade dos produtos. Por outro lado, não foi realizada uma busca destes alimentos em comércio privado, particularmente supermercados, o que pode ser considerado uma limitação do estudo, pois não se sabe o quanto esses equipamentos podem suprir a falta dos equipamentos públicos nas regiões menos abastecidas.

A verificação da distribuição espacial dos equipamentos públicos de venda de FLV em Curitiba demonstrou as desigualdades de oferta de alimentos, que são considerados importantes para a saúde, verificando que além das diferenças na oferta de alimentos, também existem iniquidades na qualidade dos equipamentos de acordo com a renda das regionais.

Curitiba é um município com alto IDH, com bons indicadores sociais e sanitários. Portanto, faz-se necessário melhor conhecimento dos fatores relatados neste estudo por parte dos gestores do município, para que se possa melhorar as políticas públicas relacionadas à alimentação e assim reduzir as diferenças encontradas, e promover o acesso a FLV e o direito à alimentação adequada da população, particularmente aquela que reside em regiões de menor condição socioeconômica.

\section{Colaboradores}

AD Cruz e Silva contribuiu no delineamento da pesquisa, interpretação dos dados e na redação do artigo. AR Silva participou na análise e interpretação dos dados e na revisão final do artigo e DA Hofelmann contribuiu na concepção e delineamento da pesquisa, análise e interpretação dos dados e revisão crítica do artigo. 


\section{Financiamento}

O presente trabalho foi realizado com apoio da Coordenação de Aperfeiçoamento de Pessoal de Nível Superior - Brasil (CAPES), Código de Financiamento 001.

\section{Referências}

1. World Health Organization (WHO). Obesity and overweight: keys facts [Internet]. Geneva: WHO; 2018. [cited 2019 Out 28]. Available from: https://www. who.int/news-room/fact-sheets/detail/obesity-and -overweight

2. Martins APB, Levy RB, Claro RM, Moubarac JC, Monteiro CA. Increased contribution of ultra-processed food products in the Brazilian diet (1987-2009). Rev Saude Publica 2013; 47(4):656-665.

3. Brasil. Ministério da Saúde (MS). Guia Alimentar para a População Brasileira 2014. Brasília: MS; 2014.

4. Brasil. Ministério da Saúde (MS). Secretaria de Vigilancia em Saúde. VIGITEL BRASIL 2018 [Internet]. Brasília: MS; 2018. 152 p. [acessado 2019 out 25]. Acessado em: http://portal.saude.gov.br/portal/arquivos/pdf/vigitel_2010_preliminar_web.pdf

5. Menezes MC, Costa BVL, Oliveira CDL, Lopes ACS. Local food environment and fruit and vegetable consumption: An ecological study. Prev Med Reports 2017; 5:13-20.

6. Instituto Brasileiro de Geografia e Estatística (IBGE). Índice de Desenvolvimento Humano Municipal - Cidades e Estados [Internet]. [acessado 2019 out 25]. Disponível em: https://www.ibge.gov.br/cidades-e-estados

7. Figueira TR, Lopes ACS, Modena CM. Barreiras e fatores promotores do consumo de frutas e hortaliças entre usuários do Programa Academia da Saúde TT - Promoters and barriers to fruit and vegetable consumption among Health Academy Program\&\#039;s users. Rev nutr 2016; 29(1):85-95.

8. Lopes ACS, Menezes MC, Araújo ML. O ambiente alimentar e o acesso a frutas e hortaliças: "Uma metrópole em perspectiva”. Saúde Soc 2017; 26(3):764-773.

9. Tarricone Garcia M, Vaz Franco J, Gasparini Araújo Costa C, Bógus CM. Acesso à frutas e hortaliças em áreas periféricas da região metropolitana de São Paulo. DEMETRA Aliment Nutr Saúde 2018; 13(2):427446.

10. Fortes MF, Borges CA, Cabral W, Miranda D, Jaime PC. Mapeando as desigualdades socioeconômicas na distribuição do comércio varejista local. 2018;45-58.

11. Diez Roux A, Mair C. Neighborhoods and Health. Ann N Y Acad Sci 2010; 1186:125-145.

12. Robinson P, Dominguez F, Teklehaimanot S, Lee M, Brown A, Goodchild M. Does distance decay modelling of supermarket accessibility predict fruit and vegetable intake by individuals in a large metropolitan area? J Heal Care Poor Underserved 2013; 24(10):172185.

13. Macintyre S. Deprivation amplification revisited; or, is it always true that poorer places have poorer access to resources for healthy diets and physical activity? IJBNPA 2007; 11(291):51-55.

14. RedeSAN, FAURGS, UFRGS, MDS. Equipamentos Públicos de Segurança Alimentar e Nutricional: Experiências e aprendizados a partir de municípios. Porto Alegre; 2011.

15. Costa BVL, Freitas PP, Menezes MC, Guimarães LMF, Ferreira LF, Alves MSC, Lopes ACS. Ambiente alimentar: validação de método de mensuração e caracterização em território com o Programa Academia da Saúde. Cad Saude Publica 2018; 34(9):e00168817. 
16. Jaime PC, Duran AC, Sarti FM, Lock K. Investigating environmental determinants of diet, physical activity, and overweight among adults in Sao Paulo, Brazil. $J$ Urban Heal 2011; 88(3):567-581.

17. Pessoa MC, Mendes LL, Gomes CS, Martins PA, Velasquez-Melendez G. Food environment and fruit and vegetable intake in a urban population: a multilevel analysis. BMC Public Health 2015; 15(1):1-8.

18. Araújo AM, Ribeiro EM. Feiras do Vale: o destino de excedentes produtivos em feiras livres do Jequitinhonha, Minas Gerais. Rev Adm Roraima 2017; 7(2):221244.

19. Pereira VG, Brito TP, Pereira SB. A Feira-Livre como importante mercado para a Agricultura Familiar em Conceição do Mato Dentro (MG). Rev Cien Educ Desenvolv Hum 2017; 10(20):67-78.

20. Maluf RS. Painel sobre experiências significativas de ação local de abastecimento alimentar. 1999; 43.

21. Borges CA, Claro RM, Martins APB, Villar BS. Quanto custa para as famílias de baixa renda obterem uma dieta saudável no Brasil? Cad Saude Publica 2015; 31(1):137-148.

22. Correa EN, Padez CMP, Abreu ÂH, Vasconcelos FAG. Geographic and socioeconomic distribution of food vendors: a case study of a municipality in the Southern Brazil. Cad Saude Publica 2017;33(2):1-14.

23. Bezerra TA, Olinda RA, Pedraza DF. Insegurança alimentar no Brasil segundo diferentes cenários sociodemográficos. Cien Saude Colet 2017;22(2):637-651.

24. Organização das Nações Unidas (ONU). Atlas do Desenvolvimento Humano no Brasil nas Regiões Metropolitanas Brasileiras. Brasília; 2014.

25. Prefeitura Municipal de Curitiba. Programa Sacolão da Família [Internet]. Secretaria Municipal de Abastecimento; 2019. [acessado 2019 out 25]. Disponível em: http://www.curitiba.pr.gov.br/conteudo/sacolao -smab/247

26. Duran AC. Ambiente alimentar urbano em São Paulo, Brasil: avaliação, desigualdades e associação com consumo alimentar [tese]. São Paulo: Universidade de São Paulo; 2013.

27. Silverman BW. Density estimation for statistics and data analysis. Nova York: Chapman \& Hall/CRC; 1998.

28. Bodor J, Rose D, Farley T, Al E. Neighbourhood fruit and vegetable availability and consumption: the role of small food stores in an urban environment. Public Health Nutr 2008; 11:413-420.

29. Moore LV, Diez Roux AV, Nettleton JA, Jacobs DR, Franco M. Fast-Food consumption, diet quality, and neighborhood exposure to fast food. Am J Epidemiol 2009; 170(1):29-36.

30. Yang Y, Diez Roux AV, Auchincloss A, Rodriguez D, Brown D. Exploring walking differences by socioeconomic status using a spatial agent-based model. Heal Place 2012; 18(1):96-99.

31. Carvalho MS, Souza-Santos R. Análise de dados espaciais em saúde pública: métodos, problemas, perspectivas. Cad Saude Publica 2005; 21(2):361-378.

32. Skalinski LM, Costa MCN, Teixeira MGL. Contribuições da análise espacial para a compreensão da dinâmica de transmissão da dengue: revisão integrativa. $J$ Heal Biol Sci 2019; 7(1):53-63.
33. Herforth A, Ahmed S. The food environment, its ef fects on dietary consumption, and potential for measurement within agriculture-nutrition interventions. Food Secur 2015; 7(3):505-520.

34. Trofholz AC, Tate AD, Draxten ML, Neumark-Sztainer D, Berge JM. Home food environment factors associated with the presence of fruit and vegetables at dinner: A direct observational study. Appetite 2016;96:526-532.

35. Gary-Webb TL, Bear TM, Mendez DD, Schiff MD, Keenan E, Fabio A. Evaluation of a mobile farmer's market aimed at increasing fruit and vegetable consumption in food deserts: a pilot study to determine evaluation feasibility. Heal Equity 2018; 2(1):375-783.

36. Motter AF, Vasconcelos FAG, Correa EN, Andrade DF. Retail food outlets and the association with overweight/obesity in schoolchildren from Florianópolis, Santa Catarina State, Brazil. Cad Saude Publica 2015; 31(3):620-632.

37. Santos DS, Hino AAF, Höfelmann DA. Iniquidades do ambiente construído relacionado à atividade física no entorno de escolas públicas de Curitiba, Paraná, Brasil. Cad Saude Publica 2019;35(5):e00110218.

38. Leone LA, Beth D, Ickes SB, MacGuire K, Nelson E, Smith RA, Tate DF, Ammerman AS. Attitudes toward fruit and vegetable consumption and farmers' market usage among low-income North Carolinians. J Hunger Environ Nutr 2014; 7(1):64-76.

39. Ma X, Liese AD, Bell BA, Martini L, Hibbert J, Draper C, Jones SJ. Perceived and geographic food access and food security status among households with children. Public Health Nutr 2016;19(15):2781-2788.

40. Claro RM, Monteiro CA. Renda familiar, preço de alimentos e aquisição domiciliar de frutas e hortaliças no Brasil. Rev Saude Publica 2010; 44(6):1014-1020.

41. Secretaria Municipal de Abastecimento. Prefeitura de Curitiba. $1^{\circ}$ Plano Municipal de Segurança Alimentar de Curitiba - PLAMSAN 2016/2019. 2016.

Artigo apresentado em 06/11/2019

Aprovado em 07/05/2020

Versão final apresentada em 09/05/2020

Editores chefes: Romeu Gomes, Antônio Augusto Moura da Silva 
